\title{
Hypokalemia-Induced Rhabdomyolysis by Primary Aldosteronism Coexistent With Sporadic Inclusion Body Myositis
}

\author{
Jong Ha Lee, MD, PhD ${ }^{1}$, Eunkuk Kim, MD, PhD², Sulk Chon, MD, PhD ${ }^{3}$ \\ ${ }^{1}$ Department of Physical Medicine and Rehabilitation, Kyung Hee University School of Medicine, Seoul; \\ ${ }^{2}$ Department of Physical Education, Korea National Sport University, Seoul; \\ ${ }^{3}$ Department of Endocrinology and Metabolism, Kyung Hee University School of Medicine, Seoul, Korea
}

We describes a patient with hypokalemia-induced rhabdomyolysis due to primary aldosteronism (PA), who suffered from slowly progressive muscle weakness after laparoscopic adrenalectomy, and was later diagnosed with coexisting sporadic inclusion body myositis (sIBM). A 54-year-old Asian male presented with severe muscle weakness of both lower extremities. Laboratory findings showed profound hypokalemia, and extreme elevation of the serum creatine phosphokinase levels, suggestive of hypokalemia-induced rhabdomyolysis. Further evaluation strongly suggested PA by an aldosterone-producing adenoma, which was successfully removed surgically. However, muscle weakness slowly progressed one year after the operation and a muscle biopsy demonstrated findings consistent with sIBM. This case is the first report of hypokalemia-induced rhabdomyolysis by PA coexistent with sIBM, to the best of our knowledge.

Keywords Rhabdomyolysis, Aldosteronism, Hypokalemia, Inclusion body myositis

\section{INTRODUCTION}

Rhabdomyolysis is defined as a pathological condition of skeletal muscle cell damage leading to the release of potentially toxic intracellular materials into the blood circulation [1]. Rhabdomyolysis is caused by various conditions such as muscle diseases, intense exercise, dehy-

Received July 24, 2014; Accepted December 4, 2014

Corresponding author: Eunkuk Kim

Department of Physical Education, Korea National Sport University, 1239 Yangjae-daero, Songpa-gu, Seoul 05541, Korea

Tel: +82-2-410-6830, Fax: +82-2-410-6719, E-mail: lkimg@knsu.ac.kr

(c) This is an open-access article distributed under the terms of the Creative Commons Attribution Non-Commercial License (http://creativecommons. org/licenses/by-nc/4.0) which permits unrestricted noncommercial use, distribution, and reproduction in any medium, provided the original work is properly cited.

Copyright (C) 2015 by Korean Academy of Rehabilitation Medicine dration, toxins, infections, various drugs and electrolyte disorders [2]. Hypokalemia induced by primary aldosteronism (PA) is a chronic process and most patients can tolerate the symptoms of malaise, muscular weakness and fatigability. However, under some extreme conditions, PA can induce excessive potassium excretion followed by rhabdomyolysis [1].

Sporadic inclusion body myositis (sIBM) is the most common myopathy after 50 years of age, with an estimated incidence and prevalence of 7.9 and 70 cases per million inhabitants, respectively, adjusted for sex and age to the 2000 U.S. Census population [3], although sIBM is considered to be even less common among Asian and African-American people [4].

This case report describes a patient with hypokalemiainduced rhabdomyolysis secondary to PA, who suffered 
from persistent muscle weakness after laparoscopic adrenalectomy, and was later diagnosed with coincident sIBM.

\section{CASE REPORT}

A 54-year-old Asian male, of height $175 \mathrm{~cm}$, weight 85 $\mathrm{kg}$, and body mass index $27.8 \mathrm{~kg} / \mathrm{m}^{2}$, presented to our department with severe muscle weakness of both lower extremities. Two weeks before presentation, he had an episode of weakness in both hands that resolved on the next day. He also reported generalized muscle weakness that had persisted for $>4$ years, which he attributed to a previous traffic accident. He denied any acute infections, trauma or intoxication. His medical history revealed a history of resistant hypertension for about 5 years that was treated with felodipine $5 \mathrm{mg}$, losartan $100 \mathrm{mg}$ and atenolol $50 \mathrm{mg}$. A month prior to presentation, he was started on thiazide $25 \mathrm{mg}$ to achieve better blood pressure control. On admission, he was conscious, with blood pressure of $170 / 100 \mathrm{mmHg}$, pulse rate of 72 beats/ min and body temperature of $36.5^{\circ} \mathrm{C}$. The initial physical examination revealed motor weakness in all limbs, especially in both lower extremities with hip flexion strength using the Medical Research Council (MRC) scale of grade 4 , knee extension grade 3 and ankle dorsiflexion/plantar flexion grade 0 . Although he complained of having trouble making fists, the MRC scale of muscle strength throughout both upper extremities was $>$ grade 4 . There were no apparent sensory symptoms or signs, and deep tendon reflexes were normoactive. The laboratory examination on admission showed extremely low serum potassium $(2.0 \mathrm{mmol} / \mathrm{L})$ and elevated creatine phosphokinase (CPK) (2,982 IU/L; reference range, 60-220 IU/L), myoglobin ( $688.9 \mathrm{ng} / \mathrm{mL}$; reference range, $17.4-105.7 \mathrm{ng} /$ $\mathrm{mL}$ ) and lactate dehydrogenase (LDH) (811 IU/L; reference range, 218-472 IU/L) levels. Urinary potassium by a spot urine check was $25.4 \mathrm{mmol} / \mathrm{L}$, potassium excretion was $75.2 \mathrm{mmol} / \mathrm{L}$ and the transtubular potassium concentration gradient was 10.36 in a 24 -hour urine collection test. Arterial blood gas analysis revealed metabolic alkalosis (serum $\mathrm{pH}=7.46, \mathrm{PaCO}_{2}=42.6 \mathrm{mmHg}, \mathrm{PaO}_{2}=63.3$ $\mathrm{mmHg}, \mathrm{HCO}_{3}=29.9 \mathrm{mEq} / \mathrm{L}$, and $\mathrm{SaO}_{2}$ at $\left.93.3 \%\right)$. Electrocardiogram showed a decline in $\mathrm{T}$-wave amplitudes and abnormal U-waves with a regular sinus rhythm. Magnetic resonance imaging of the cervical and lumbar spine was performed to determine whether there was a pathological lesion at the spinal level that could account for the patient's severe muscle weakness. This showed central and left paracentral and inferior disc extrusion at L5-S1, ossification of the posterior longitudinal ligament at C5-6 and central and left paracentral disc protrusion at C5-6 and C6-7, but these findings could not explain the generalized severe muscle weakness by the patient (Fig. 1).

Based on the above clinical findings, the patient was first diagnosed with severe hypokalemia and rhabdomyolysis. The thiazide was discontinued due to the possibility of drug-induced hypokalemia, and oral and intravenous potassium supplementation was initiated. The severe muscle weakness and the serum CPK levels improved over the course of 2 weeks. However, the generalized muscle weakness persisted, especially in both lower extremities and hands, and the serum potassium levels remained low (around $3.0 \mathrm{mmol} / \mathrm{L}$ ). Subsequent electro-
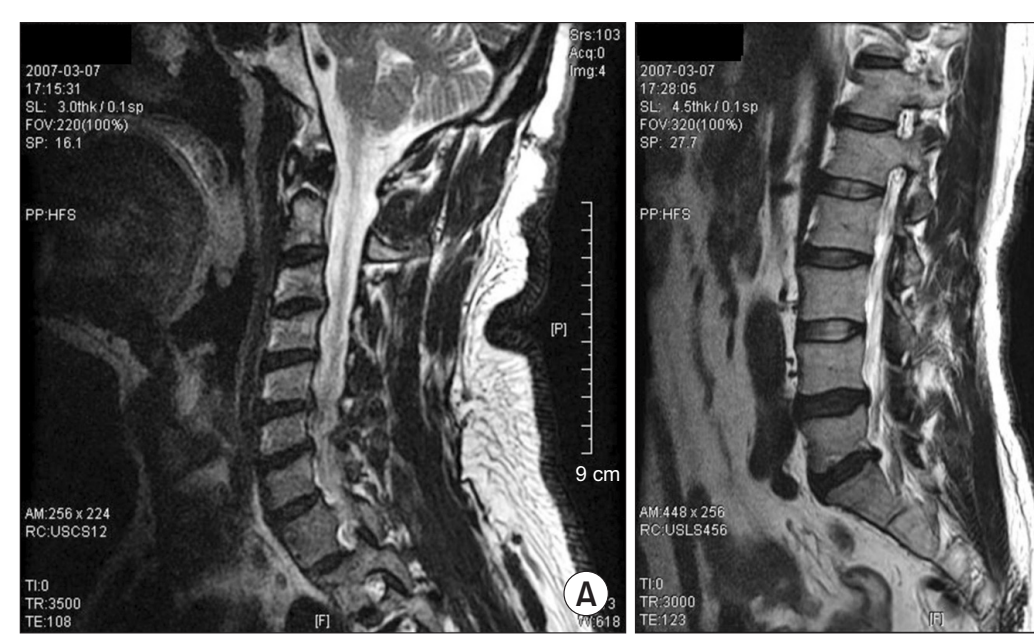

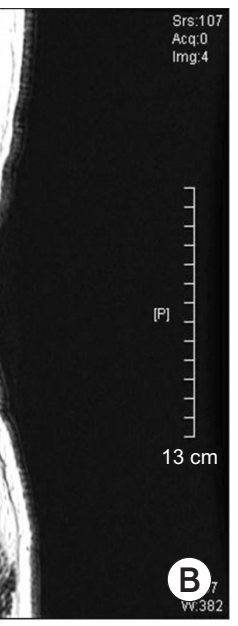

Fig. 1. Magnetic resonance imaging findings of the (A) cervical and (B) lumbar spines. Central and left paracentral and inferior disc extrusion at L5-S1, ossification of the posterior longitudinal ligament at C5-6 and central and left paracentral disc protrusion at C5-6 and C6-7 were shown. 
Table 1. Nerve conduction study findings of the patient

\begin{tabular}{|c|c|c|c|c|}
\hline Stimulation nerve & Recording site & Latency (ms) & Amplitude $(\boldsymbol{\mu V})$ & $\mathrm{CV}(\mathrm{m} / \mathrm{s})$ \\
\hline \multicolumn{5}{|l|}{ Motor nerve conduction study } \\
\hline Left median nerve & & & & 46.6 \\
\hline Wrist & APB & 3.95 & $7,195.0$ & \\
\hline Elbow & APB & 9.10 & $6,236.7$ & \\
\hline Left ulnar nerve & & & & 60.7 \\
\hline Wrist & $\mathrm{ADM}$ & 3.65 & $6,290.0$ & \\
\hline Elbow & $\mathrm{ADM}$ & 8.10 & $5,838.0$ & \\
\hline \multicolumn{5}{|l|}{ Left peroneal nerve } \\
\hline Ankle & EDB & 5.45 & 58.3 & \\
\hline Fibular head & EDB & ND & ND & \\
\hline Left tibial nerve & & & & 42.9 \\
\hline Ankle & $\mathrm{AH}$ & 4.20 & 228.3 & \\
\hline Knee & $\mathrm{AH}$ & 13.3 & 156.7 & \\
\hline \multicolumn{5}{|l|}{ Sensory nerve conduction study } \\
\hline Left median nerve & Ankle & 3.70 & 43.9 & 37.8 \\
\hline Left ulnar nerve & Ankle & 3.65 & 26.5 & 38.4 \\
\hline Left superficial peroneal nerve & Ankle & $\mathrm{ND}$ & ND & - \\
\hline Left sural nerve & Ankle & 3.90 & 4.6 & 35.9 \\
\hline
\end{tabular}

$\mathrm{APB}$, abductor pollicis brevis muscle; ADM, abductor digiti minimi muscle; EDB, extensor digitorum brevis muscle; $\mathrm{AH}$, abductor hallucis muscle; CV, conduction velocity; ND, not detected.

Table 2. Needle electromyographic findings of the patient

\begin{tabular}{|c|c|c|c|c|c|c|c|}
\hline \multirow{2}{*}{ Muscle } & \multicolumn{3}{|c|}{ Spontaneous } & \multicolumn{3}{|c|}{ MUAPs } & \multirow{2}{*}{$\begin{array}{c}\text { Recruitment } \\
\text { pattern }\end{array}$} \\
\hline & IA & Fib & PSW & Poly & Amp & Dur & \\
\hline Lt. APB & Normal & None & None & Normal & Large & Prolonged & $\downarrow$ \\
\hline Lt. FCR & $1+$ & $1+$ & $1+$ & + & Large & Prolonged & $\downarrow$ \\
\hline Lt. FCU & $1+$ & None & $1+$ & + & Large & Prolonged & $\downarrow$ \\
\hline Lt. ECR & Normal & None & None & + & Large & Prolonged & $\downarrow$ \\
\hline Lt. Deltoid & Normal & None & None & Normal & Large & Prolonged & $\downarrow$ \\
\hline Lt. Cer PS & Normal & None & None & Normal & Normal & Normal & Not checkable \\
\hline Lt. AH & Normal & None & None & \multicolumn{4}{|c|}{ Not checkable } \\
\hline Lt. EDB & Normal & None & None & \multicolumn{4}{|c|}{ Not checkable } \\
\hline Lt. TA & $2+$ & None & $2+$ & \multicolumn{4}{|c|}{ Not checkable } \\
\hline Lt. MG & Normal & None & None & \multicolumn{4}{|c|}{ Not checkable } \\
\hline Lt. VM & Normal & None & None & + & Large & Prolonged & $\downarrow$ \\
\hline Lt. Gmax & Normal & None & None & + & Normal & Prolonged & $\downarrow$ \\
\hline Lt. Lum PS & Normal & None & None & Normal & Normal & Normal & Not checkable \\
\hline
\end{tabular}

MUAPs, motor unit action potentials; IA, insertion activity; Fib, fibrillation; PSW, positive sharp wave; Poly, polyphasic potential; Amp, amplitude; Dur, duration; Lt., left; APB, abductor pollicis brevis muscle; FCR, flexor carpi radialis muscle; FCU, flexor carpi ulnaris muscle; ECR, extensor carpi radialis muscle; Cer PS, cervical paraspinal muscle; AH, abductor hallucis muscle; EDB, extensor digitorum brevis muscle; TA, tibialis anterior muscle; MG, medial gastrocnemius muscle; VM, vastus medialis muscle; Gmax, gluteus maximus muscle; Lum PS, lumbar paraspinal muscle; $\downarrow$, reduced. 
diagnostic studies demonstrated neuropathic patterns. Nerve conduction studies revealed a axonal peripheral neuropathy (motor and sensory conduction studies of peroneal nerves were not evoked and the other motor and sensory nerve conduction studies showed small amplitudes, delayed latencies and decreased velocities) (Table 1), and needle electromyography showed spontaneous activities in the flexor carpi radialis, flexor carpi ulnaris, and tibialis anterior muscles, and motor unit action potentials (MUAPs) were not evoked at the abductor hallucis, extensor digitorum brevis, medial gatrocnemius and tibialis anterior muscles and large amplitude polyphasic MUAPs were observed in all the other examined muscles (Table 2). Persistent hypokalemia with excessive urinary potassium loss and resistant hypertension raised the suspicion of PA; therefore, an abdominal computed tomography (CT) was done and showed no abnormal findings at the time.

At this point, the patient was referred to the department of endocrinology for further evaluation and management. Endocrinologic evaluations revealed suppressed plasma rennin activity (PRA; $0.3 \mathrm{ng} / \mathrm{mL} / \mathrm{hr}$; reference range, $0.8-1.36 \mathrm{ng} / \mathrm{mL} / \mathrm{hr}$ ), increased plasma aldosterone concentration (PAC; $37.5 \mathrm{ng} / \mathrm{dL}$; reference range, 5.0-19.4 $\mathrm{ng} / \mathrm{dL}$ ) and an extremely high PAC/PRA ratio (125 ng/ $\mathrm{dL}$ per $\mathrm{ng} / \mathrm{mL} / \mathrm{hr}$; reference range, less than $20-25 \mathrm{ng} /$ $\mathrm{dL}$ per $\mathrm{ng} / \mathrm{mL} / \mathrm{hr}$ ). A furosemide stimulation test did not demonstrate an increase in PAC after 2 hours of standing, indicating that the most likely cause of PA was an aldosterone-producing adenoma (APA). A repeat abdominal
CT scan was performed, which showed a small, circumscribed, hypo-attenuated right adrenal mass measuring $1.5 \mathrm{~cm} \times 1.0 \mathrm{~cm}$ in diameter (Fig. 2A). A month after admission, the patient was treated successfully by laparoscopic right adrenalectomy. The histological findings of the specimen were consistent with a functioning adrenocortical adenoma (Fig. 2B, C). The patient was diagnosed with APA resulting in severe hypokalemia and subsequent rhabdomyolysis. Two weeks after the operation, the patient's symptoms improved, the PAC and PRA reverted abruptly to normal levels, and the serum potassium and CPK levels also returned to and remained normal.

At 1 year post-operatively, the patient was normotensive and normokalemic, but still complained of generalized muscle weakness (hip flexion strength was grade 3, knee extension was grade 3, ankle dorsiflexion/plantar flexion were still grade 0 , and finger flexors were grade 3 ), which seemed to have slowly progressed to the point of impairing activities of daily living. Laboratory examination showed a normal CPK, LDH and myoglobin, although his clinical presentation appeared similar to certain types of adult-onset neuropathy or myopathy. This was grounds for the suspicion that a certain type of neuromuscular disease had been coexistent with the patient's hypokalemia-induced rhabdomyolysis caused by PA. Therefore, a muscle biopsy of the left vastus lateralis muscle was performed, which demonstrated endomysial inflammation and vacuolar degeneration consistent with sIBM (Fig. 3). Within 6 months of establishing the diagnosis and beginning rehabilitative management, he was
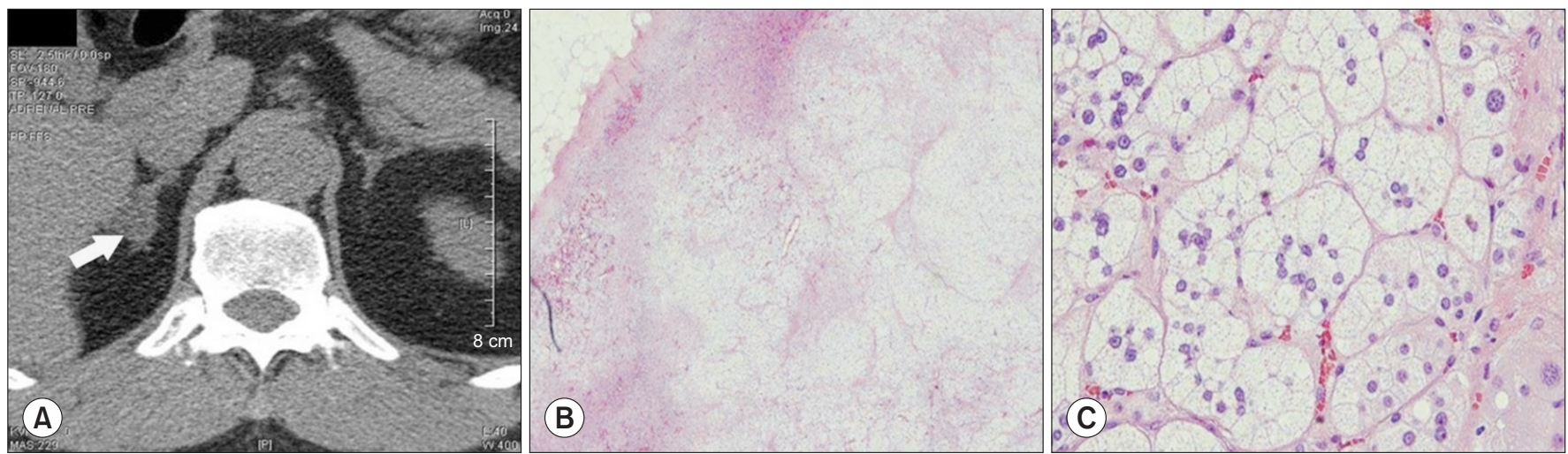

Fig. 2. (A) Adrenal computed tomography shows a $1.5 \times 1-\mathrm{cm}$-sized ovoid mass at the body of the right adrenal gland. (B, C) Microscopic findings of the resected right adrenal gland. Sections disclose portions of adrenal cortical adenoma showing a well circumscribed tumor tissue consisting of proliferation of some zona glomerulosa and mostly fasciculata-resembling cells (H\&E, B 100×, C 400×). 

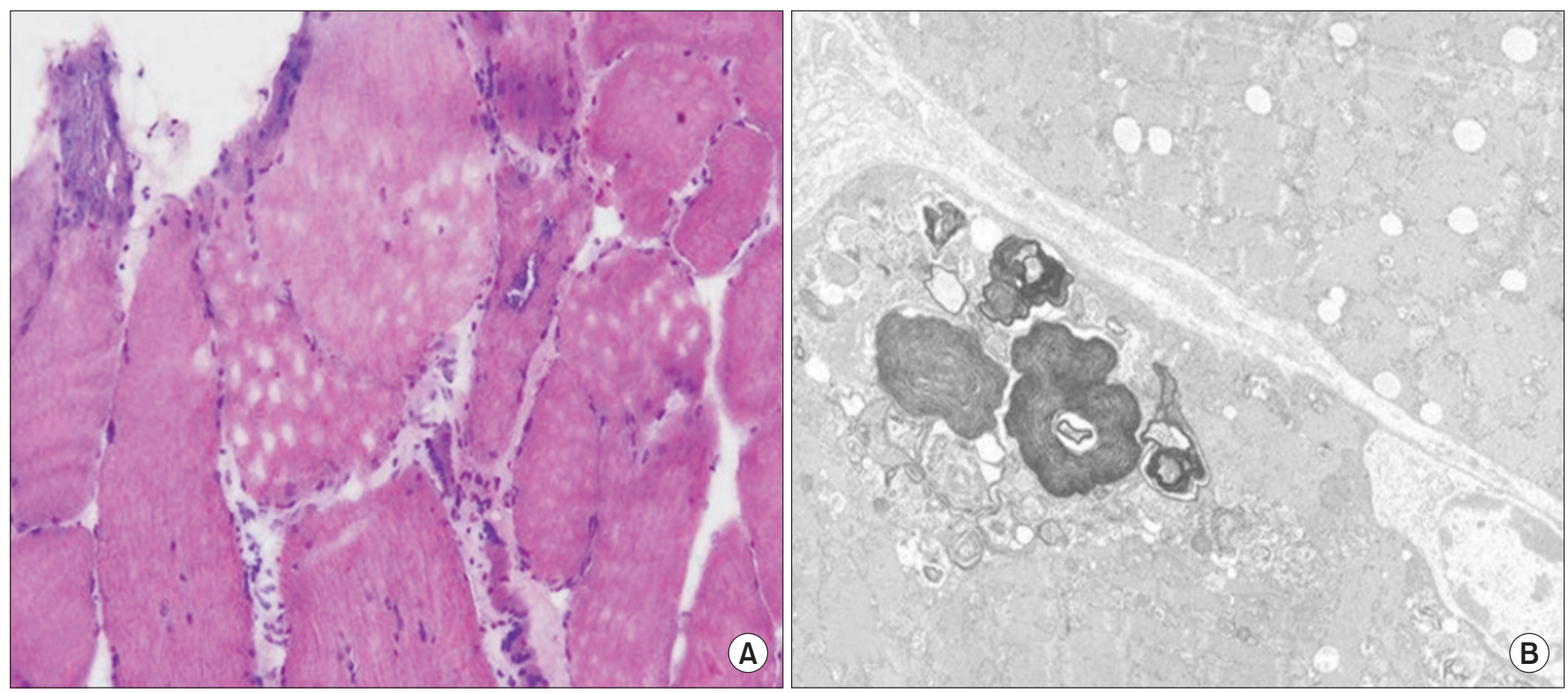

Fig. 3. (A) Light microscopy of the biopsy specimen from the left vastus lateralis shows marked size variation of myofibers; some rimmed vacuoles with basophilic stippling; round atrophic and degenerating myofibers; endomysial fibrosis and fatty change (H\&E 400×). (B) Electron microscopy shows size variation of myofibers with rarefaction of myofilaments; many myelin figure-like concentric lamellated membranous bodies and degenerated organelles in subsarcolemmal and intermyofibrillar areas (electron microscope $6000 \times$ ), consistent with inclusion body myositis.

wearing bilateral ankle-foot orthoses and using a wheelchair for outdoor ambulation.

\section{DISCUSSION}

PA is a common cause of secondary hypertension and the prevalence rates of PA currently range between $5 \%$ $10 \%$, although a true estimation is difficult because its detection is dependent on the physician's level of awareness of the disorder [2]. A recent study showed that hypokalemia was present in $45.6 \%$ of patients with resistant hypertension due to PA; in addition, PA can induce excessive potassium excretion followed by rhabdomyolysis in some extreme conditions $[1,2,5]$. The present patient was first diagnosed with rhabdomyolysis based on the findings of severe muscle weakness, high serum CPK and myoglobin levels; and the rhabdomyolysis was considered to have resulted from severe hypokalemia. Although rhabdomyolysis usually results in hyperkalemia due to the direct release of intracellular potassium into the extracellular fluid, over-excreted aldosterone induced potassium excretion into the urine, in the present patient. The cause of hypokalemia-induced rhabdomyolysis in PA is unknown, but some reports suggest that the potassium ion is a major factor mediating the rise of muscle blood flow and it is postulated that impaired potassium release from the potassium-deficient skeletal muscle in patients with PA may induce muscle injury or frank necrosis as a consequence of the relative ischemia [6,7].

Hypokalemia is one of the most frequent findings among hypertensive patients with PA [5]. However, many patients with PA are not hypokalemic [8] and PA-induced hypokalemia is usually mild and can present many years after the appearance of arterial hypertension [9]. The malaise and muscle weakness from the mild hypokalemia is usually tolerable for patients [1], but serum potassium levels may drop to very low levels in PA when vomiting or diarrhea occurs, or diuretics are used [2]. Frank rhabdomyolysis usually occurs only when serum potassium values are $<2.0 \mathrm{mmol} / \mathrm{L}$, as in the present case [1]. Goto et al. [2] reported a patient on a diuretic to achieve better blood pressure control 2 weeks prior to his admission for severe weakness in his extremities that developed shortly after a heavy bout of drinking alcohol. In this patient, profound hypokalemia was thought to be precipitated by alcohol-induced dehydration in addition to the diuretic therapy.

In our case, the patient was started on $25 \mathrm{mg}$ of a thia- 
zide for uncontrollable hypertension 1 month prior to presentation. Despite the history of resistant hypertension, longstanding muscle weakness, and a recent episode of bilateral hand weakness, he had never been screened for secondary hypertension until admission, and diuretic therapy was initiated antecedently. The initiation of the thiazide was likely to have aggravated the hypokalemia, although there were no other precipitating factors such as alcohol-induced dehydration. The severe muscle weakness of the patient appeared to be improving and the serum potassium and CPK levels normalized shortly after the diagnosis of PA by APA and its successful removal. However, the patients' muscle weakness persisted and progressed slowly during the 1 year post-operative period. Therefore, we presumed that the underlying and previously undiagnosed sIBM probably contributed as a potential precipitating factor to the development of severe rhabdomyolysis in the hypokalemic skeletal muscle, which led to the subsequent, progressive muscle weakness.

sIBM is characterized clinically by the insidious onset of slowly progressive proximal and distal weakness, which generally develops after the age of 50 years, and the presence of asymmetric, quadriceps and wrist/finger flexor weakness strongly suggests the diagnosis of sIBM [10]. Ankle dorsiflexors are also often involved at an early stage, as in the present case [3]. The slow progress of the disease may in part account for the delay in diagnosis [4], and electrodiagnostic study results suggesting an axonal peripheral neuropathy in the nerve conduction study and pseudo-neurogenic MUAPs may prevent the diagnosis of myopathy. In the present study, there was no suspicion of sIBM at first because of the sudden onset of severe muscle weakness. Moreover, sIBM, which is considered to be rare among Asians [10], was coexistent with PA associated with rhabdomyolysis due to profound hypokalemia that has also been rarely reported $[1,2]$. It is a challenge for physiatrists to suspect 2 rare coincident conditions. In the present case, the slowly progressive muscle weakness after the removal of the apparent cause of the patient's symptoms, raised the suspicion of another underlying neuromuscular condition, thus prompting the decision to perform a further evaluation (muscle biopsy) and eventually revealing coexistent sIBM. sIBM is reportedly associated with some autoimmune diseases [3]. However, there are no previous case reports on IBM associated with endocrine syndromes. Therefore, coexisting endocrinological disorder might be an extremely rare presentation of sIBM. This is the first report of hypokalemia-induced rhabdomyolysis by PA coexistent with sIBM, to the best of our knowledge.

The present case report indicates that sIBM might manifest with PA by APA of the adrenal gland, which causes severe hypokalemia manifesting as generalized weakness due to rhabdomyolysis. When evaluating hypertensive patients with generalized muscle weakness, it is important to evaluate serum electrolyte concentrations, especially serum potassium levels, and further determine the cause of hypokalemia. The present case emphasized the need for physiatrists to monitor accompanying signs and symptoms that suggest another coexisting neuromuscular condition.

\section{CONFLICT OF INTEREST}

No potential conflict of interest relevant to this article was reported.

\section{REFERENCES}

1. Wen Z, Chuanwei L, Chunyu Z, Hui H, Weimin L. Rhabdomyolysis presenting with severe hypokalemia in hypertensive patients: a case series. BMC Res Notes 2013;6:155.

2. Goto A, Takahashi Y, Kishimoto M, Minowada S, Aibe $\mathrm{H}$, Hasuo K, et al. Primary aldosteronism associated with severe rhabdomyolysis due to profound hypokalemia. Intern Med 2009;48:219-23.

3. Dimachkie MM, Barohn RJ. Inclusion body myositis. Curr Neurol Neurosci Rep 2013;13:321.

4. Greenberg SA. Inclusion body myositis. Curr Opin Rheumatol 2011;23:574-8.

5. Douma S, Petidis K, Doumas M, Papaefthimiou P, Triantafyllou A, Kartali N, et al. Prevalence of primary hyperaldosteronism in resistant hypertension: a retrospective observational study. Lancet 2008;371:1921-6.

6. Ma JT, Wang C, Lam KS, Yeung RT, Chan FL, Boey J, et al. Fifty cases of primary hyperaldosteronism in Hong Kong Chinese with a high frequency of periodic paralysis: evaluation of techniques for tumour localisation. Q J Med 1986;61:1021-37.

7. Knochel JP, Schlein EM. On the mechanism of rhab- 
domyolysis in potassium depletion. J Clin Invest 1972; 51:1750-8.

8. Mattsson C, Young WF Jr. Primary aldosteronism: diagnostic and treatment strategies. Nat Clin Pract Nephrol 2006;2:198-208.

9. Loh KC, Koay ES, Khaw MC, Emmanuel SC, Young WF
Jr. Prevalence of primary aldosteronism among Asian hypertensive patients in Singapore. J Clin Endocrinol Metab 2000;85:2854-9.

10. Amato AA, Barohn RJ. Inclusion body myositis: old and new concepts. J Neurol Neurosurg Psychiatry 2009;80:1186-93. 